\title{
The Electric Field in Irradiated Silicon Detectors ${ }^{1}$
}

\author{
L J Beattie, T J Brodbeck, A Chilingarov, G Hughes, S A McGarry, P N Ratoff \\ and T Sloan
}

Department of Physics

University of Lancaster

Lancaster LA1 4YB, UK

\begin{abstract}
The electric field distribution inside heavily irradiated silicon particle detectors is deduced using observations of $\alpha$ particle and MIP signals. In these detectors $\alpha$ particle signals are observed for both $\mathrm{p}^{+}$and $\mathrm{n}^{+}$side illumination even when the detector is only partially depleted. The observations indicate that the electric field distribution within the detector has the contribution expected from a uniform space charge, as in unirradiated detectors, together with a strong, short range, local electric field in the vicinity of the $\mathrm{p}^{+}$ electrode.

PACS: 07.77-n; 85.30.-z
\end{abstract}

Keywords; electric field,irradiated,silicon,detectors

\section{Introduction}

The behaviour of unirradiated silicon particle detectors is well understood. If they are illuminated by $\alpha$ particles, signals are observed from the junction side at all voltages whereas they are only observed from the ohmic side at voltages just below the depletion voltage when the electric field reaches a depth to which the $\alpha$ particles penetrate. In

${ }^{1}$ Submitted to Nucl.Inst. and Meths A 
contrast, irradiated detectors behave differently in a manner similar to that observed in more complicated materials such as GaAs [1].

In this paper we describe our observations of $\alpha$ particle signals for illumination from both sides of a heavily irradiated detector as well as those of minimum ionising particles (MIPs) from a $\beta$ source. We use the data to show that in such detectors there is a similar electric field pattern to that in an unirradiated detector together with an extra local electric field in the vicinity of the $\mathrm{p}^{+}$electrode. This extra field is shown to be strong and of short range.

The detector used in all the measurements described here was a standard n-type p-i-n diode of thickness $300 \mu \mathrm{m}$ irradiated by a fluence of $1.310^{14} \mathrm{~cm}^{-2}$ pions of momentum $300 \mathrm{MeV} / \mathrm{c}$. After irradiation it was left for about a week at room temperature to allow beneficial annealing [2] to take place and then it was stored at a temperature of $\sim 0^{\circ} \mathrm{C}$. The measurements described below were made at temperatures between 10 and $20^{\circ} \mathrm{C}$ taking care that the detector did not spend more than a few hours at these temperatures to avoid reverse annealing [2]. This detector is one of a sample of 11 similar diodes used for a general study of radiation damage effects in Si. The charge collection results for the detector which are reported here are similar to those observed in the other detectors [3] which were irradiated by fluences of between $1-310^{14} \mathrm{~cm}^{-2}$ pions or neutrons. Hence the data presented here can be regarded as typical of a detector subject to such irradiation fluences.

\section{Signals from $\alpha$ particles in irradiated detectors}

As is well known [4] after heavy irradiation the initial n-type silicon becomes p-type. Therefore the junction moves to the $\mathrm{n}^{+}$side of the detector and the depletion region should develop from this side. To investigate this, irradiated detectors were tested with $\alpha$ particles. Surprisingly, signals were observed for $\mathrm{p}^{+}$side illumination even at voltages well below the depletion voltage. Fig 1 shows the $\alpha$ particle signal measured in terms of the charge collection efficiency (CCE) in the irradiated Si detector for $\alpha$ particles illuminating the $\mathrm{n}^{+}$and $\mathrm{p}^{+}$sides. The CCE is the ratio of the charge observed in the detector to that observed in the same detector before irradiation. 


\section{$2.1 \alpha$ particle signal from the $\mathbf{n}^{+}$side}

Fig 1a shows the measured CCE for $\mathrm{n}^{+}$side illumination by $\alpha$ particles as a function of the square root of the bias voltage, $U_{b}$, together with a linear fit to the data up to the depletion voltage, $U_{d}$, which is $209 \mathrm{~V}$ in this case. The lowest three voltages were omitted from this fit since the drift field in the diode becomes so low that the plasma effect [5] begins to influence the results. It can be seen that the linear fit gives a good representation of the data. The simplest explanation of this is based on the assumption that the undepleted bulk acts as an insulator for fast electric signals produced by the induced charge on the detector electrodes $[6,3]$. Since, in this case, Ramo's theorem [7] predicts that the signal is proportional to the width of the depletion region, $x_{d}$, for short range $(\sim 20 \mu \mathrm{m}) \alpha$ particles and we observe that the signal is proportional to $\sqrt{U_{b}}$, it follows that $x_{d}$ should be proportional to $\sqrt{U_{b}}$. Hence we conclude that the depletion region grows from the $\mathrm{n}^{+}$side and its width varies as the square root of the bias voltage as would be expected for a detector with uniform space charge.

However, the CCE does not reach $100 \%$ at the depletion voltage and the loss is ascribed to charge trapping. This is expected to be constant with bias below the depletion voltage since the trapping of the charge during the drift through the depleted region should be approximately independent of the thickness of this region. This can be understood from the compensation between the increasing drift distance $\left(x_{d} \sim \sqrt{U_{b}}\right)$ and increasing drift velocity of the carriers with increasing bias. The characteristic drift time $t_{d}=x_{d}^{2} / \mu U_{b}$ then remains the same for all values of $U_{b}$, hence keeping constant the trapping which is proportional to $e^{-t_{d} / \tau}$. Here $\mu$ is the carrier mobility and $\tau$ is the carrier lifetime due to trapping in the material.

\section{$2.2 \alpha$ particle signal from the $\mathrm{p}^{+}$side}

Fig. $1 \mathrm{~b}$ shows the measured CCE from $\alpha$ particles illuminating the $\mathrm{p}^{+}$side of the detector, as a function of the bias voltage. In contrast to unirradiated detectors the signal from the $\mathrm{p}^{+}$side for $\alpha$ particles (Fig 1b) grows smoothly with bias rather than suddenly appearing when the detector becomes almost fully depleted. The smooth curve in Fig $1 \mathrm{~b}$ is a fit to the data based on the model which is described in Section 5.

The unexpected observation of $\alpha$ particle signals from the $\mathrm{p}^{+}$side in irradiated de- 
tectors shows that there must be an electric field present in such detectors at this side. To investigate the strength of this field the time of arrival of the signals from the $\mathrm{p}^{+}$side was compared with that from the $\mathrm{n}^{+}$side. If the field on the $\mathrm{p}^{+}$side is weak the reduced carrier velocity will cause a significant delay in the arrival of the signal from this side. Conversely, if the field is strong the delay will be small.

The delay was measured by comparing the differences of the signal arrival times and the $\alpha$ particle impact times for $\mathrm{p}^{+}$and $\mathrm{n}^{+}$side $\alpha$ particle illumination of the detector. The time delay between the $\alpha$ particle impact and the signal arrival was measured using $\alpha-\gamma$ coincidences from an ${ }_{95}^{241} \mathrm{Am}$ source. The impact time of the $\alpha$ particle was obtained by detecting the coincident $\gamma$ ray in a scintillation counter. The $\gamma$ signal was used to start a Time to Amplitude Converter and the $\alpha$ signal to stop it, so that the output amplitude gave a measure of the relative time delay between the $\alpha$ particle impact time as signalled by the $\gamma$ ray and the ionisation arrival time. The time spectra were measured for both $\mathrm{p}^{+}$and $\mathrm{n}^{+}$side $\alpha$ particle illumination.

Figure 2 shows a typical time spectrum. The events at large delay time arise from random coincidences which populate the whole time range uniformly. The peak corresponds to the real coincidences. The exponential rise up to the peak at small times is due to the fact that the excited ${ }_{93}^{237} \mathrm{~Np}$ daughter nucleus from ${ }_{95}^{241} \mathrm{Am}$ decay is an isomeric transition with a mean lifetime of $\sim 30 \mathrm{~ns}$. Hence there is an exponential time distribution of the start times obtained from the $\gamma$ ray with a sharp fall to the random coincidence count level. This sharp fall corresponds to the earliest arrival time of the $\gamma$ signal. All the time spectra taken showed similar shapes to that in Fig 2. The sharpness of the fall to the random background shows that there is little time smearing of the ionisation as it passes through the detector and this fall time was similar for all the measurements. To obtain the delay time of the ionisation in the detector a fit of a functional form was performed to each spectrum. This function consisted of a flat (random) background together with an exponential rise up to a fixed time, which was a free parameter, with a gaussian fall at times greater than this fixed time. The half maximum point on this gaussian fall gave a measure of the average relative delay of the ionisation arrival time in the detector.

Time slewing in the discriminator connected to the detector occurred because of the finite risetime $(\sim 60 \mathrm{~ns})$ of the detector's amplifier. A correction for this risetime was made by measuring the time slewing for different amplitude pulses from an unirradiated 
detector. The corrected arrival times were then measured for both $\mathrm{p}^{+}$and $\mathrm{n}^{+}$illumination and the difference was taken to obtain the ionisation drift time.

Fig 3 shows the difference between the signal arrival times for $\alpha$ particles impinging on the $\mathrm{p}^{+}$and the $\mathrm{n}^{+}$sides as a function of the normalised depletion layer thickness given by $d=\sqrt{U_{b} / U_{d}}$, where $U_{d}$ is the depletion voltage. The delay time is short $(\sim 7 \mathrm{~ns})$ and independent of the bias voltage (or depletion layer thickness) within the errors. The plasma delay has been measured to be $\sim 27 \mathrm{~ns} / E$ with $E$ in $\mathrm{kV} / \mathrm{cm}[8]$. For the measured delay time to be so short the electric field in the vicinity of the $\mathrm{p}^{+}$electrode must be of the order of several $\mathrm{kV} / \mathrm{cm}$ i.e. similar in strength to the field in the bulk of the detector. Hence, there must be a rather strong electric field in the vicinity of the $\mathrm{p}^{+}$electrode of the detector.

\section{Signals from MIPs in irradiated detectors}

Signals from MIPs in the irradiated detectors were measured using a collimated $\beta$ source and a system of scintillation counters to define particles which traverse the whole detector as described in [9]. Fig 4 shows the MIP CCE as a function of the detector bias voltage together with a linear fit to the data up to the depletion voltage. This fit gives a good representation of the data showing that the CCE increases linearly with bias with an intercept at zero voltage of about $2 \%$.

Such a linear behaviour can be understood as follows. The deposited ionisation energy varies linearly with the depletion width and the induced signal per unit ionisation energy also varies linearly with the depletion width from Ramo's theorem [7], as discussed in section 2.1. Hence the signal from MIPs which traverse the whole detector will vary as the product of the two effects i.e. as the square of the depletion width. The behaviour of the detector for $\mathrm{n}^{+}$illumination by $\alpha$ particles shows that this depletion width varies as the square root of the bias voltage (see Fig 1a), hence the signal from MIPs will vary linearly with bias voltage, as observed. We argue that the intercept at zero voltage can arise from the electric field near the $\mathrm{p}^{+}$side (see below). 


\section{The Electric Field Pattern in Irradiated Detectors}

A picture emerges of the electric field distribution in the irradiated detectors from all the above information. This field is similar to that for a uniform space charge in the depletion region falling to near zero at the edge of the depletion region. This is necessary to explain the linear behaviour of the MIP CCE with bias and the linear behaviour of the $\mathrm{n}^{+} \alpha$ particle CCE with the square root of the bias voltage. However, in order to explain the observation of $\alpha$ signals from the $\mathrm{p}^{+}$side which are not significantly delayed there must be a strong local field in the vicinity of the electrode on this side. Such a strong local field which is generated by the material would also explain the small, non-zero intercept of the CCE for MIPs extrapolated to zero bias and also the fact that the $\alpha$ particle CCE from the $\mathrm{p}^{+}$side extrapolates to a non-zero value at zero bias voltage. The fact that these intercepts are quite small shows, from Ramo's theorem [7], that the field has a short range and that it is present at all biases causing a current to flow locally which gives signals even at very small bias voltage.

Fig. 5 shows a schematic diagram of the electric field pattern in the detector which we deduce from these observations. The width of the region of the electric field adjacent to the $\mathrm{p}^{+}$side must be comparable to the $\alpha$ particle range $(\sim 20 \mu \mathrm{m})$.

\section{A Model for the $\alpha$ particle CCE from the $\mathbf{p}^{+}$side.}

Assuming that the electric field pattern follows the form described in the previous section we can develop a model to describe the observed $\alpha$ particle CCEs for illumination from the $\mathrm{p}^{+}$side for voltages below the depletion voltage, $U_{d}$ (see Fig $1 \mathrm{~b}$ ). We assume the following parameterisation;

$$
C C E=E_{0}+E_{1} d A(d)
$$

where $d$ is the normalised thickness of the depleted bulk, given by $d=\sqrt{U_{b} / U_{d}}, A(d)$ is a function describing the attenuation due to trapping of the charge during the drift through the non depleted part of the bulk and $E_{0}$ and $E_{1}$ are constants. The constant $E_{0}$ can be interpreted as the part of the CCE produced by the movement of the ionisation before it reaches the depleted region of the detector. Assuming that this is dominated by the initial movement of the ionisation in the strong, local field near the $\mathrm{p}^{+}$electrode then it 
can be regarded as a constant independent of $d$. The constant $E_{1}$ is interpreted as the normalised charge averaged over the drift distance in the depleted area. Following the same arguments as those used in section 2.1 one can assume that $E_{1}$ is independent of $U_{b}$. The function $A(d)$ is assumed to be an exponential function of the relative thickness of the undepleted layer, $x=1-d$, with an attenuation length $a$. Applying the obvious boundary condition that $A(x=0)=1$ and assuming zero signal from ionisation in the depletion region at $U_{b}=0$ so that $A(x=1)=0$, one obtains for $A(x)$;

$$
A(x)=\frac{\exp (-x / a)-\exp (-1 / a)}{1-\exp (-1 / a)}
$$

from which $A(d)$ is derived.

The smooth curve in Fig $1 b$ represents a fit of this simple model to the $\alpha$ data from the $\mathrm{p}^{+}$side with $E_{0}, E_{1}$ and $a$ as free parameters. The model fits the data well suggesting that the assumptions made in deriving it are probably reasonable.

The fit with $E_{0}=0$ gives a noticeably worse agreement with the data than when $E_{0}$ is allowed to remain finite. This indicates that the initial movement of the ionisation in the detector must happen even at very low bias. Hence the strong electric field near the $\mathrm{p}^{+}$electrode occurs for all bias voltages. The physical origin of this strong field could be the existence of a thin non-inverted n-type layer adjacent to the $\mathrm{p}^{+}$electrode (as already noticed some time ago[10,11]) which leads to a "transistor-like" structure $\mathrm{p}\left(\right.$ bulk)-n- $\mathrm{p}^{+}$. For any polarity of the applied bias such a "transistor" switches to the "punch-through" mode as soon as the bias voltage can provide the necessary voltage drop across the structure. If one assumes that the thickness of this non-inverted n-layer is a few microns and it has a voltage drop across it of a few volts it gives the same field in the layer as the average field in a $300 \mu \mathrm{m}$ thick detector biased by $300 \mathrm{~V}$. Such a field can naturally produce the value of $E_{0}$ of a few percent as observed in the data.

To reproduce the smooth growth of the CCE from the $\mathrm{p}^{+}$side with $U_{b}$ the model described by equation (1) requires also the existence of a non-zero electric field in the part of the bulk between the depleted area and the high field layer near the $\mathrm{p}^{+}$electrode (see Fig.5). This should allow electrons released near the $\mathrm{p}^{+}$surface to drift to the depleted area in a finite time from where they are quickly transported to the $\mathrm{n}^{+}$electrode. The high mobility of the electrons drifting from the $\mathrm{p}^{+}$electrode makes this penetration fast enough 
for detection of the charge within the $70 \mathrm{~ns}$ integration time used in our electronics.

\section{Conclusions}

Signals from $\alpha$ particles and MIPs have been investigated in heavily irradiated Silicon detectors. The data indicate that the electric field falls to near zero from the $\mathrm{n}^{+}$side just as it should for a uniform space charge in the depleted region. However, the irradiation causes a strong, short range, local field in the vicinity of the $\mathrm{p}^{+}$electrode so that $\alpha$ particle signals from this side are observed even when the detectors are far from being fully depleted. A simple parameterisation of the CCE for this case based on this electric field pattern has been developed.

\section{Acknowledgements}

We thank S M Holt for his excellent technical assistance. We also thank G Lutz, B K Jones, R Richter and K F Smith for helpful and stimulating discussion.

\section{References}

[1] B K Jones et al., Nucl.Instr.and Meth. A395(1997)81.

[2] H-J.Ziock et al., Nucl.Instr.and Meth. A342(1994)96.

[3] L.J.Beattie et al., "Charge collection in heavily irradiated silicon diodes", ATLAS Internal Note INDET-No-171, 16 June 1997 (to be submitted to Nucl.Intstr.and Meth. A)

[4] F.Lemeilleur et al., IEEE Trans.Nucl.Sci. NS-39(1992)551.

[5] R.N.Williams and E.M.Lawson, Nucl.Instr.and Meth. 120(1974)261.

[6] Z.Li and H.W.Kraner IEEE Trans.Nucl.Sci. NS-38(1991)244.

[7] S.Ramo IRE 27(1939)584.

[8] L.J.Beattie et al., "Carrier lifetimes in heavily irradiated Si diodes", (to be submitted to Nucl.Instr. and Meth. A) 
[9] A.Chilingarov and T.Sloan, Proc.of the III Int.Workshop on GaAs and Related Compounds, San Miniato, March 21-24 1995, World Scientific, p.52.

[10] Z.Li and H.W.Kraner, IEEE Trans.Nucl.Sci. NS-39(1992)577.

[11] R.Wunstorf, Ph.D thesis, DESY FH1K-92-01. 


\section{Figure Captions}

- Fig 1. a) Charge Collection Effiency for $\alpha$ particles as a function of $\sqrt{U_{b}}$ for $\mathrm{n}^{+}$side illumination. The linear fit is discussed in the text. b) Charge Collection Efficiency for $\alpha$ particles as a function of $U_{b}$ for $\mathrm{p}^{+}$side illumination. The smooth curve is the result of the fit to the data of the model described in the text.

- Fig 2. Time difference spectrum between the $\gamma$ ray arrival and the arrival of the ionisation from the $\alpha$ particle. The smooth curve is the result of the fit described in the text.

- Fig 3. Difference in the arrival times (in ns) of the $\alpha$ particle signals from the $\mathrm{p}^{+}$ and $\mathrm{n}^{+}$sides as a function of the normalised depletion width $d=\sqrt{U_{b} / U_{d}}$.

- Fig 4. Measured CCE for MIPs as a function of the bias voltage. The straight line is the linear fit to the data discussed in the text.

- Fig 5. A schematic diagram of the electric field pattern inside an irradiated silicon detector deduced from this work. 

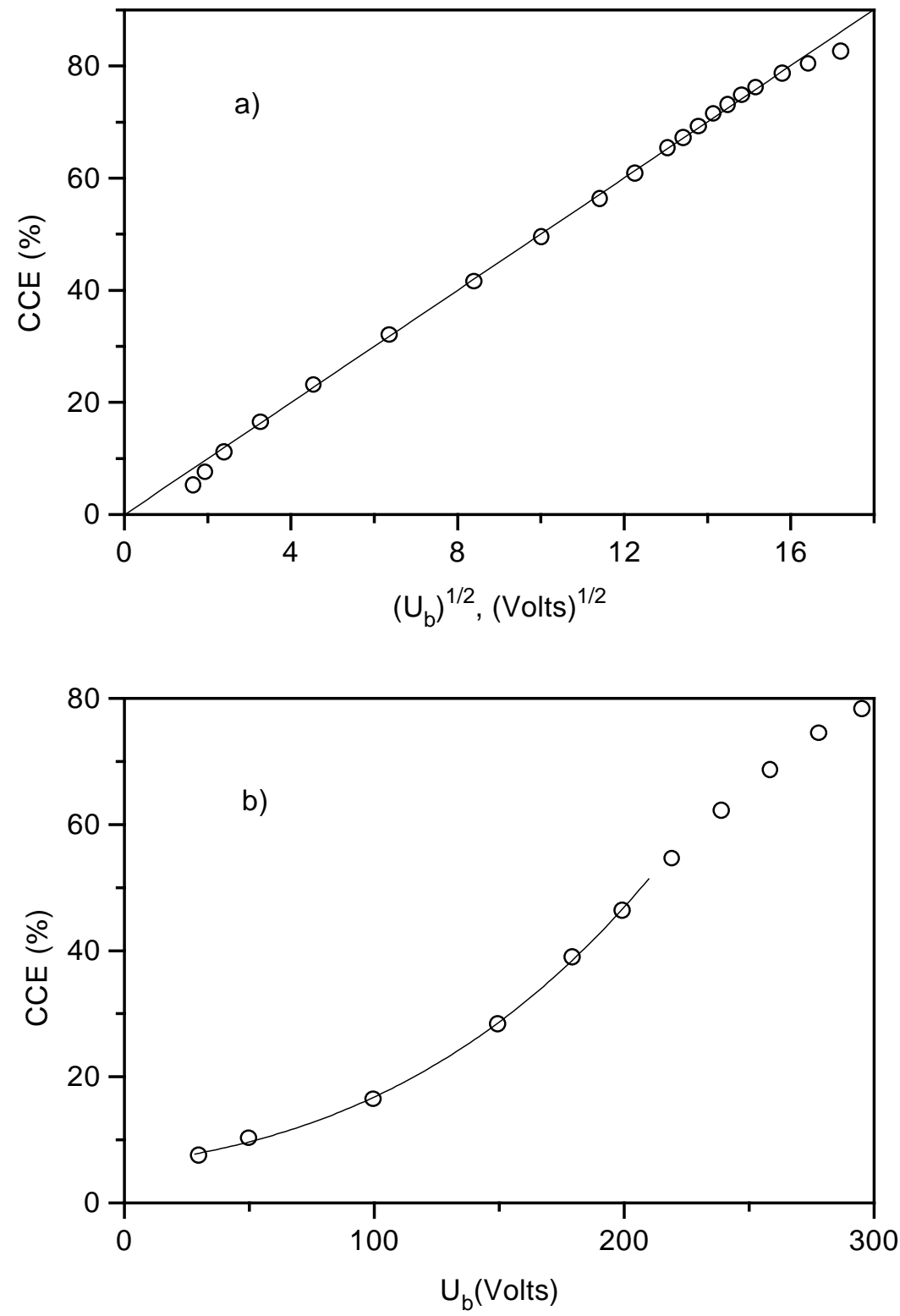

Fig 1 


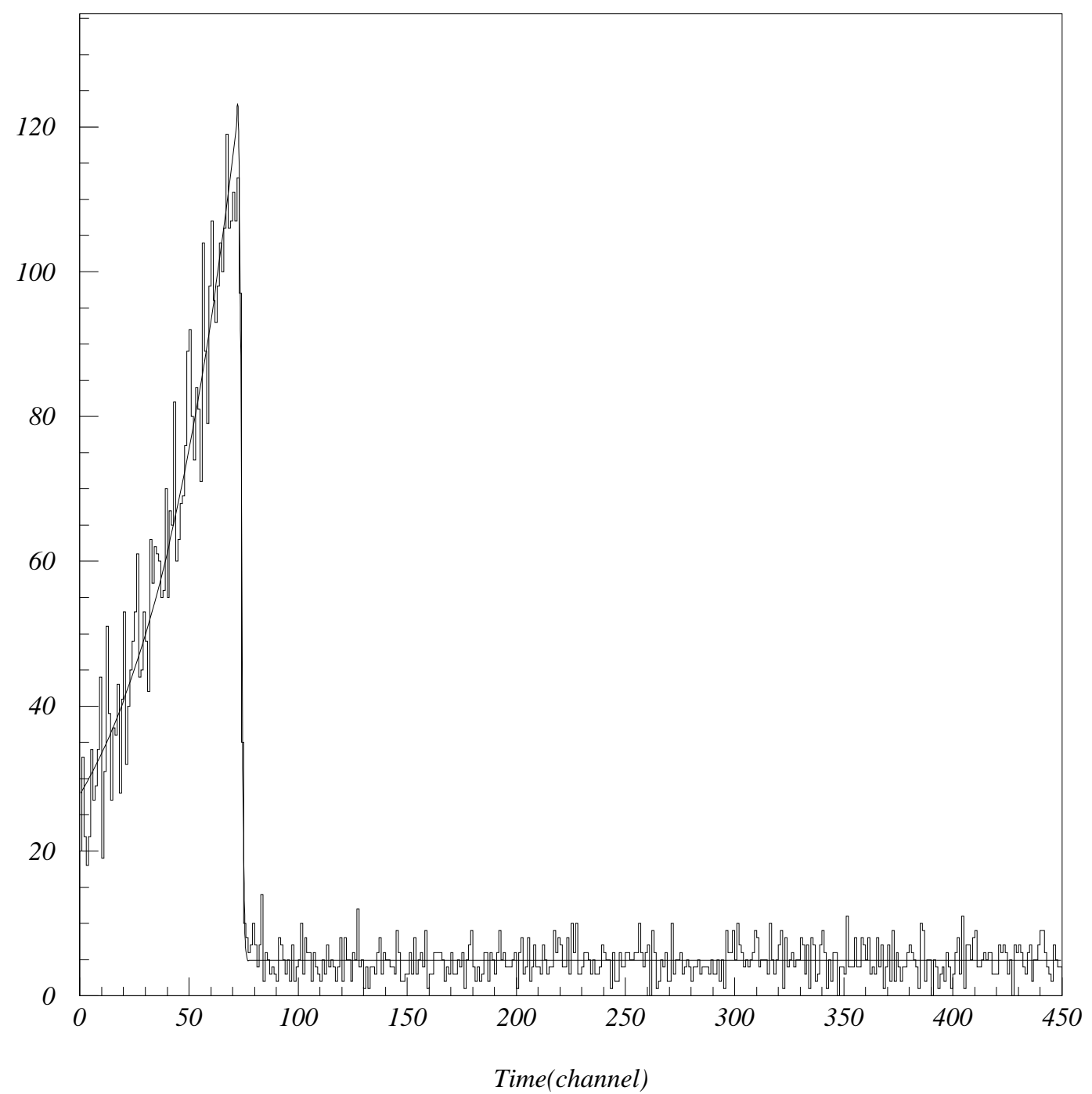

Fig 2 
Front - back time differences

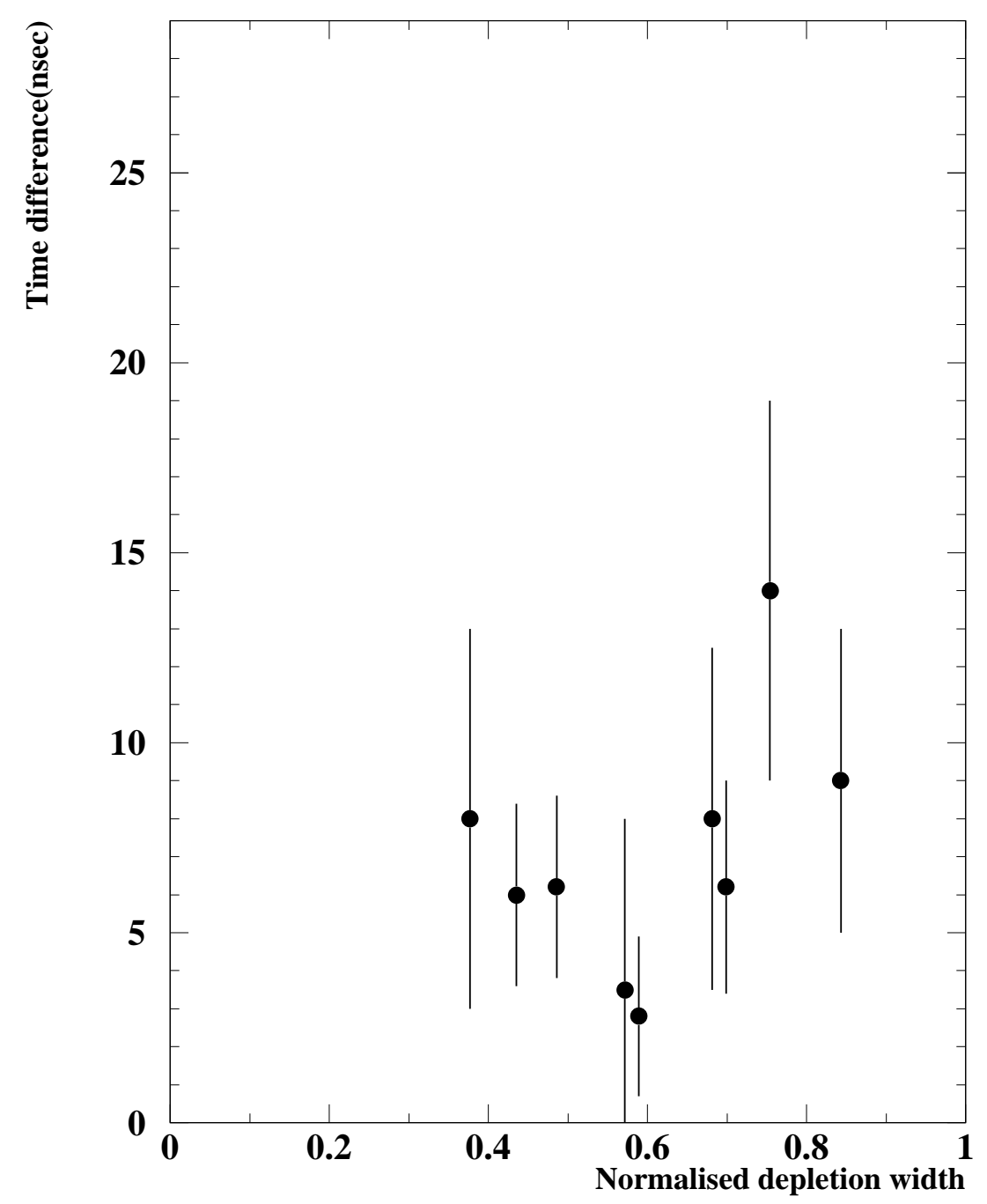

Fig 3 


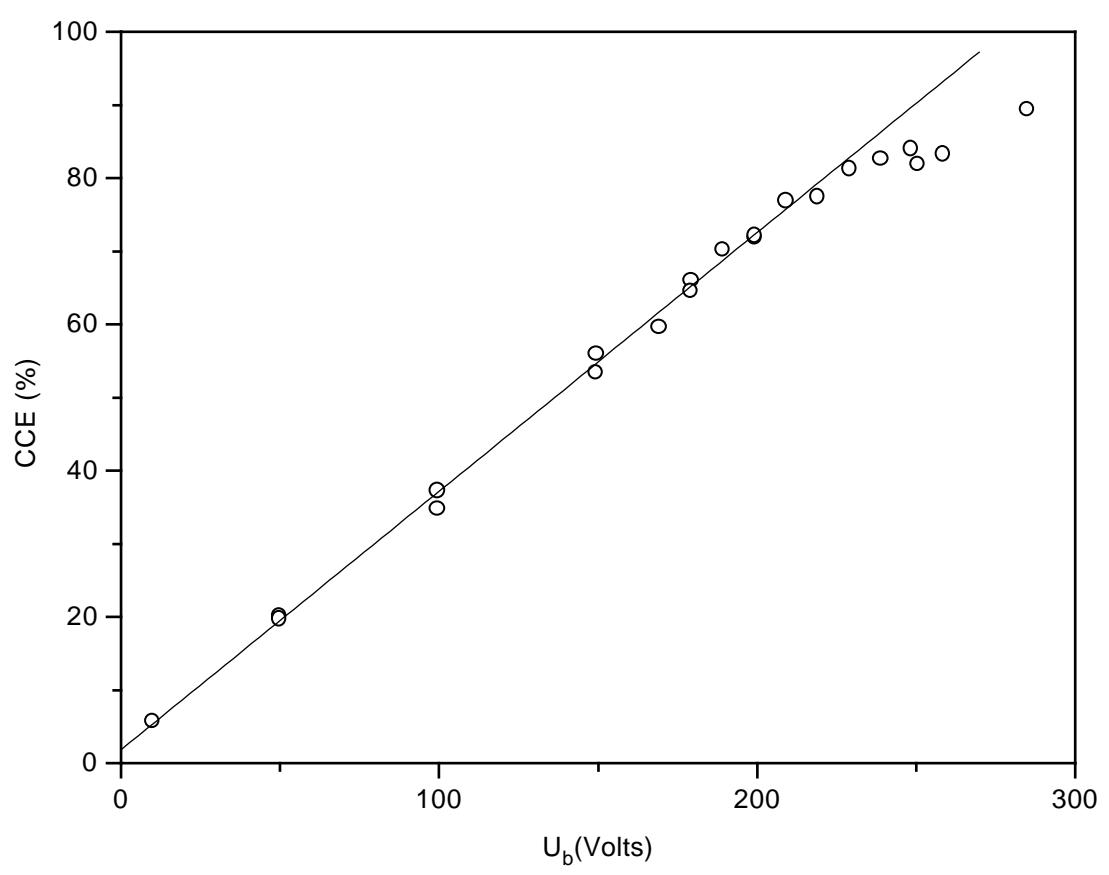

Fig 4 


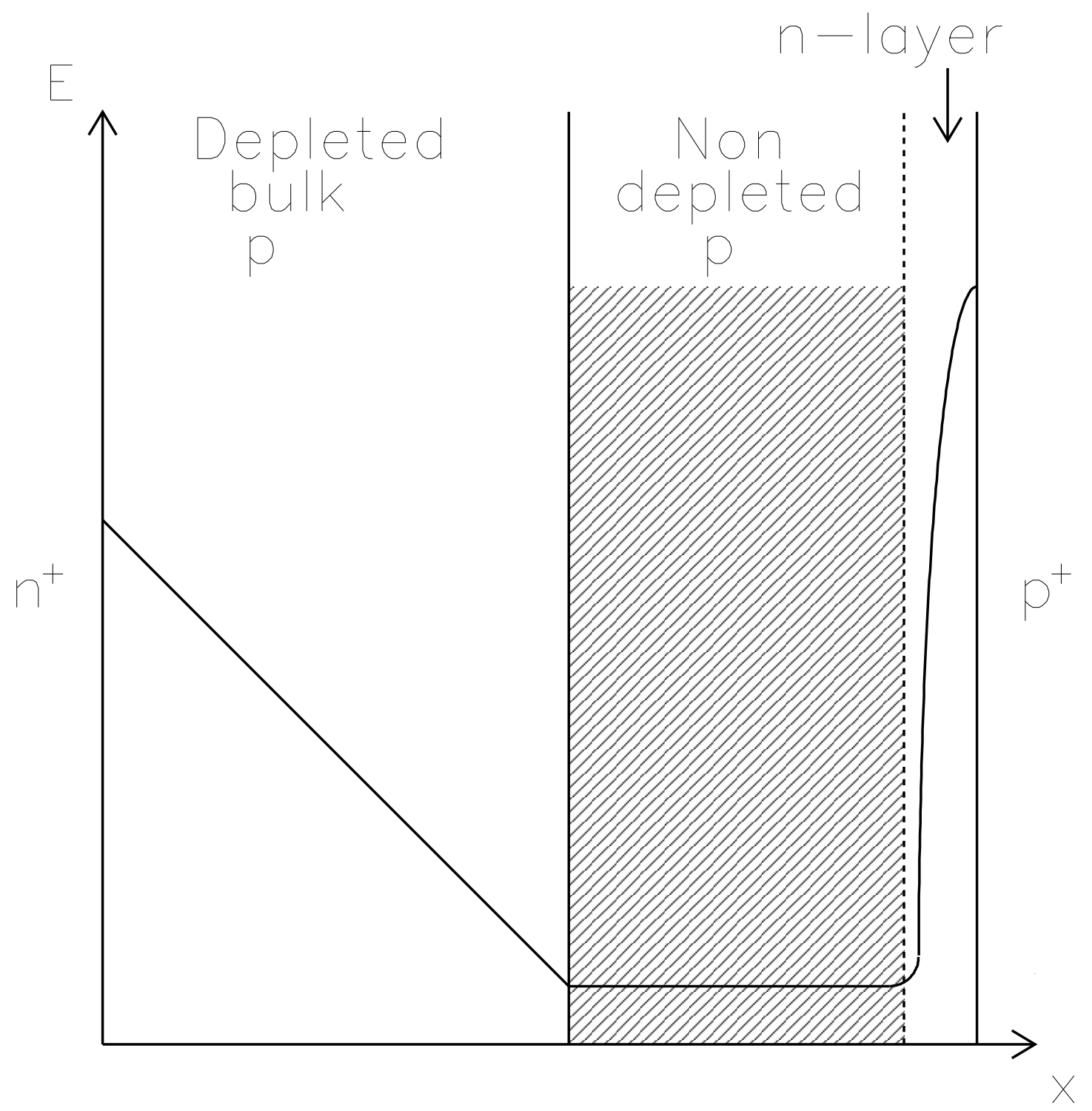

Fig 5 